\title{
Interactive comment on "Accounting for Carbon and Nitrogen interactions in the Global Terrestrial Ecosystem Model ORCHIDEE (trunk version, rev 4999): multi-scale evaluation of gross primary production" by Nicolas Vuichard et al.
}

Anonymous Referee \#2

Received and published: 26 March 2019

(A) General comments :

This paper describes the evaluation of a revised version of the ORCHIDEE model, incorporating representations of the carbon $(\mathrm{C})$ and nitrogen $(\mathrm{N})$ (and water) interactions. This paper comes 9 years after the initial publication of a first version of a $\mathrm{C}-\mathrm{N}$ version of the ORCHIDEE model (Zaehle \& Friend, 2010; hereafter ZF10). one already published by ZF10, with several modifications (listed from P3L30 ("Page 3 
Line 30") to L4L11 and on P5L4-9). These modifications are mostly not mathematically described in the paper.

Different from ZF10 that evaluated fluxes simulated over a set of European forests, this paper provides an evaluation of the revised ORCHIDEE over GPP data acquired across the globe (using both Fluxnet data and a machine-learning product predicting GPP across the globe "MTE-GPP"). After this initial evaluation, the paper presents sensitivity analyses (SA) aiming at inferring the role of simulated C-N coupling on the centennial dynamic of simulated GPP.

When evaluating a revised version of a model, one needs two references: (1) groundtruth data and (2) a previous version of the model from which the one we are evaluating has been developed. Both are mandatory to provide a thorough evaluation of a revised version of a model, and conclude as whether or not the developments have indeed improved the model.

As regards ground-truth data:

- the model is evaluated against GPP time series. This is indeed an important flux, for which the model needs be evaluated. However, we are here dealing with a coupling of $\mathrm{C}$ and $\mathrm{N}$ cycles in the model. Evaluating the model against $\mathrm{C}$ flux data is clearly not enough. I know that $\mathrm{N}$ data are much less common than $\mathrm{C}$ data (e.g. Vicca et al. 2018), but the effort has already been made in earlier versions of ORCHIDEE (see ZF10 for instance). Hence I expect at least a minimal evaluation of this new version against some $\mathrm{N}$ data;

- the $\mathrm{N}$ cycle also impacts respiration. Since the Fluxnet data include both daytime and nighttime (i.e. respiration) fluxes, I see no good reason for the authors not to evaluate the model ability to simulate respiration fluxes;

- since part of the sensitivity analysis implies simulated transpiration fluxes, I also expect to see some comparison of simulated evapotranspiration against flux tower data.

Printer-friendly version

Discussion paper 
To this respect, P3L7 is misleading stating that the paper includes a "evaluation of simulated gross carbon uptake and transpiration by plants.". I see no evaluation against transpiration data in the paper.

As regards comparison with previous versions of the model: when reading the paper, I cannot evaluate how the model modifications affected the model prediction accuracy. As said above, there are two groups of modifications listed by the authors: group 1 (p. 3-4 of the manuscript) seems to be overlooked by the authors, while group 2 (p. 5: modifications in the photosynthesis scheme and in the photosynthesis- $\mathrm{N}$ coupling) appear more important (i.e. the authors refer to them later in the paper). If the authors think group 2 would significantly impact the simulations, I expect to see a model comparison confronting simulations from a former (e.g. O-CN?) and the current model version. Since two main modifications are mentioned (modification of the photosynthesis scheme and modification of the photosynthesis- $N$ coupling), I expect to see how both independently impact the model output.

Based on these two points (partial model evaluation against ground-truth data and lack of comparison with model previous versions to evaluate the impact of model modifications), I think the paper in its current version is not ready for publication.

(B) Additional comments:

P2L18, replace "is plentiful" by "is non-limiting provided adequate mineral nutrition in the future,"

P2L21, replace "will" by "would"

P3L7 "thorough"

P5L30 eq. 2: on which data were the parameters fitted ? On GPP data? These parameters are very sensitive, please be precise.

P8: How were equations 11 and 12 parameterized? Fitted on which data?

Printer-friendly version

Discussion paper 
P11L15 : replace "ran" by "run" (past participle form, several occurrences throughout the text).

P11L22 "feeding back"

P14L25: "differences in the simulated mineralisation and plant Nitrogen uptake (not shown)." Is certaintly very informative (probably more that forcing Ndep time series as appears on Fig. 2), that's a pity we cannot see that.

P18L4 says that one of the modifications of the model is "the maximum Rubisco activity-limited carboxylation rate is a direct function of the leaf nitrogen content (Kattge et al., 2009)"... well that was already the case in OCN (see eq. 4 of model appendix description in ZF10).

Table 1: Where do these values come from? Parameter values are for CNleaf,min and CNleaf,max are not documented.

Fig2c, TeDBF: How does it occur that $\mathrm{C} / \mathrm{N}$ either decreases or increases from June to December in TeDBF ? in $\mathrm{NH}$, it should increase (leaf $\mathrm{N}$ decreases : $\mathrm{N}$ resorption while $\mathrm{C}$ remains about constant).

(C) References:

Vicca, S., Stocker, B. D., Reed, S., Wieder, W. R., Bahn, M., Fay, P. A., ... \& Rebel, K. T. (2018). Using research networks to create the comprehensive datasets needed to assess nutrient availability as a key determinant of terrestrial carbon cycling. Environmental Research Letters, 13(12), 125006.

Zaehle, S., \& Friend, A. D. (2010). Carbon and nitrogen cycle dynamics in the OâĂŘCN land surface model: 1. Model description, siteâĂŘscale evaluation, and sensitivity to parameter estimates. Global Biogeochemical Cycles, 24(1).

Printer-friendly version

Discussion paper

Interactive comment on Geosci. Model Dev. Discuss., https://doi.org/10.5194/gmd-2018-261, 2018. 